\title{
Determination of Dopamine Using Screen-printed Carbon Electrode Modified by PEDOT-MWCNTs double-layer Composites
}

Zhiqiang Wei, Yile Hu, Qingqing Tu, Shimeng Cui, Yiran Li, Yu Gan, Guilong Li, Hui Yang*, Sanqiang $L i^{*}$

College of Basic Medical, Henan University of Science and Technology, Luoyang, 471023, Henan, China.

*E-mail: yanghui7761@ 163.com, sanqiangli2001@163.com

doi: $10.20964 / 2021.06 .23$

Received: 3 February 2021 / Accepted: 24 March 2021 / Published: 30 April 2021

The poly(3,4-ethylenedioxythiophene) (PEDOT)-MWCNTs composites with double-layer structure $\left((\mathrm{MWCNTS} / \mathrm{PEDOT})_{2}\right.$-PEDOT) were prepared on the commercially available screen-printed carbon electrode (SPCE) by a simple solution coating approach. Due to the unique double-layer structure and the good synergistic effect of PEDOT and MWCNTs, the (MWCNTs/PEDOT) $)_{2}$-PEDOT modified SPCE ((MWCNTs/PEDOT) $)_{2}$-PEDOT/SPCE) demonstrated excellent electrocatalytic properties for the oxidation of dopamine (DA). Under the optimal experimental conditions, the oxidation peak currents of DA increased linearly with two concentration intervals over the range of $0.02-0.95 \mu \mathrm{M}$ and 0.0 .95 $15 \mu \mathrm{M}$, respectively. And the detection limit $(\mathrm{S} / \mathrm{N}=3)$ was $0.01 \mu \mathrm{M}$. Furthermore, the proposed sensor was applied to the electrochemical determination of DA in Human serum samples, and the recoveries for DA were from $98.3 \%$ to $101.5 \%$ with relative standard deviation (RSD) between $2.7 \%$ and $3.8 \%$. It showed great application prospects in pharmaceutical analysis.

Keywords: Dopamine; Screen-printed Carbon Electrode; PEDOT-MWCNTs; Double-layer Composites

\section{$\underline{\text { FULL TEXT }}$}

(C) 2021 The Authors. Published by ESG (www.electrochemsci.org). This article is an open access article distributed under the terms and conditions of the Creative Commons Attribution license (http://creativecommons.org/licenses/by/4.0/). 